\title{
Dynamic Performance Analysis of Capture and Separation Process for a Microsatellite Docking Device
}

\author{
DAI Ye ${ }^{1 *}$, ZHANG Yuan ${ }^{1}$, LI Wen-juan ${ }^{2}$ and TANG Wen-ming ${ }^{1}$ \\ ${ }^{1}$ School of Mechanical \& Power Engineering, Harbin University of Science and \\ Technology, Heilongjiang Harbin 150080, China \\ ${ }^{2}$ School of Electrical \& Electronic Engineering, Harbin University of Science and \\ Technology, Heilongjiang Harbin 150080, China \\ *daiye312@163.com
}

\begin{abstract}
Aiming at the problem of short lifetime and high cost in the on-orbit Servicing technology, a new type of automatic autonomous probe-cone docking mechanism used for microsatellite docking is proposed which can realize on-orbit special services. The structure of the mini docking mechanism is provided according to the design indices, and a virtual prototype model of the mini-type universal docking system is established by using MATLAB software. The dynamics modeling of the docking process is presented based on the virtual work principle. The simplified dynamic analysis model during the capture process is established. The dynamic characteristic analysis of the catching process is completed to research the influence of the key parameters, and the virtual prototype model of the docking device is established through ADAMS for further verifying the proposed design. The analysis shows that this mechanism has the good capacity of capture and separation process and energy absorption to reduce the impact during the docking process. And the simulation results meet the design demands of mini-type universal autonomous docking mechanism.
\end{abstract}

Keywords: docking device; numerical simulation; capture process; dynamic analysis

\section{Introduction}

With the high-speed development of modern aviation industry, it asks for higher requirements of on-orbit servicing technology, hence, the automatic autonomous docking devices gradually occupies the increasingly important position[1-3]. The purpose of developing microsatellite docking mechanism is to make the satellite maintain in an effective and stable orbit as long as possible in order to prolong the on-orbit lifetime and reduce the alteration frequency. The mini-type universal docking mechanism is an essential equipment for realizing the docking process of tracing satellite and space station or tracing satellite and other one, which is widely used in astronautics, aviation, national defense[4]. The mini-type universal autonomous docking mechanism is the key equipments for achieving the autonomous satellite docking, which have been widely used in the aerospace, aviation, defense and other fields.

The capability of docking, refueling, repairing and updating microsatellites by using automatic autonomous vehicles will be of significance critical value for the design and operation of several space systems in the near feature. Automatic docking capability was successfully tested by many institutions, such as NASDA in Japan, European Space Agency, NASA in USA etc. The microsatellite docking mechanism would greatly expand the application range and increase the flexibility of the satellite[5-7]. This has been a hot research topic all over the world. Flight tests have been performed using the soft impact docking mechanism of the ETS-VII satellite of Japan. An A5DS docking mechanism used 
for the Orbital Express plan and a COMET self-powered docking test are all under development.

The docking process involves the dynamics and kinematics of the spacecrafts. So the dynamic simulation, analysis and design of the structural parameters and the driving parameters, design of buffer parameter and checking of the structural strength have to be included[8-10]. As a kind of universal equipment, the dynamic characteristics of its key parts could directly affect the reliability of satellite docking system. Hence, accurate and reasonable kinetic model of the docking process must be established to verify the design of the docking mechanism, and the simulation and experiments for analysis of the reliability of unlocking and separation in the docking process must be carried out.

\section{Program Analysis of System Integration}

\subsection{Structural Components of Docking Device}

The designed docking mechanism is composed of a passive docking mechanism (PDM) and an active docking mechanism (ADM) that are respectively mounted on the active and the passive spacecrafts. The design of this automatic autonomous mini probe-cone docking mechanism should mainly consider the some principles, such as having simple structure, compact layout, and convenient installation; Light weight of the docking mechanism within the $10-20 \mathrm{~kg}$ weight; safe and reliable docking, having the capacity of energy absorption to reduce the impact during the docking process as much as possible, even if in case there is a big attitude deviation, they also can successfully capture, and so on.

Table 1. Main Composition and Basic Functions of Docking Mechanism

\begin{tabular}{ccl}
\hline Component & \multicolumn{1}{c}{ Name } & \multicolumn{1}{c}{ Basic functions } \\
\hline \multirow{3}{*}{ PDM } & $\begin{array}{c}\text { Trigger mechanism } \\
\text { Positioning } \\
\text { component } \\
\text { Connecting } \\
\text { mechanism }\end{array}$ & $\begin{array}{l}\text { Triggering internal components of capture mechanism } \\
\text { Eliminating pitch angle deviation and yaw attitude } \\
\text { angle deviation } \\
\text { Realizing connection with passively docked satellite }\end{array}$ \\
& Automatic capture & Completing capture task and bearing buffering power \\
& Driving capture mechanism to make chain actions \\
& Realizing forward extension and retraction of probing \\
& Dody cap & Driving expansion link and locking mechanism \\
& Locking mechanism & Locking ADM and PDM \\
Buffer damping & Realizing flexible collision and docking of mechanism \\
& mechanism & Completing fuel conveying task \\
& Injection mechanism & Completing measurement of buffer parameter and \\
& Measuring system & attitude \\
\hline
\end{tabular}

The passive docking mechanism includes a trigger mechanism, a positioning component and a connecting mechanism, and the active docking mechanism mainly comprises an automatic capture mechanism, a driving component, and a locking mechanism, a buffer damping mechanism, an injection mechanism and a measuring system. The main components and the basic functions of the docking mechanism are listed in Table.1.

\subsection{Design Principle of Docking Device}

In this paper, the capture and separation process simulation of the docking device is the main analysis tasks. Therefore, the following design principles during the capture and 
separation process of the automatic autonomous mini probe-cone docking device should be mainly considered:

(1) During the capture phase, the capture mechanism is driven by a stepper motor to extend forward until to the limit position. After the capture mechanism is triggered, the automatic positioning of lap joint between the active docking mechanism and the passive docking mechanism can be realized. Then the stepper motor is driven backward such that the active spacecraft gradually approaches the passive spacecraft. And the motor and a reduction gear are driven, and four locks on the active docking mechanism rotate $90^{\circ}$ to the same direction through the locking mechanism. Hence the locking of the active and passive spacecrafts can be realized.

(2) In the separation process, the capture mechanism is driven to go backward such that the positioning component that has been ejected out is retracted; then the motor and the reduction gear are driven to make the four locks rotate 90 degrees forward or backward for unlocking, reduce the flight speed of the active spacecraft and finally realize the separation of the two spacecrafts.

\section{Dynamic Analysis Model Considering the Impact Effects}

The research purpose of this paper is to analyze the dynamic characteristic in the capture phase of the prone-cone microsatellite docking mechanism. The main tasks considered are to establish and simplify dynamic analysis model during the capture process. In order to simply the calculation of collision contact point and collision contact force, the telescopic rod on the active docking mechanism is simplified to be a straight line segment. According to the symmetry in the structure of docking mechanism, the contact problem can be simplified into a plane. According to the structure of the docking mechanism, the whole dynamic analysis model can be simplified as shown in Fig.1.

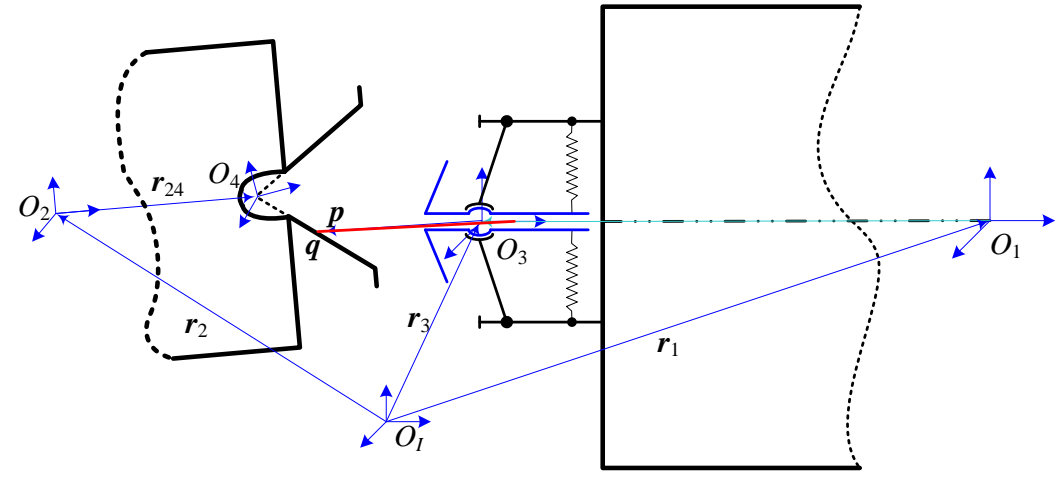

Figure 1. Simplified Model of Dynamic Analysis

In the dynamic analysis during docking process, the main concern is the relative value of various physical quantities, such as the relative displacement and the relative angular velocity between the docking spacecrafts. The coordinate system used in the process of docking is defined as shown in Fig.1: 1) Coordinate system on fixed connection of mass center of the active docking spacecraft: $O_{1} X_{1} Y_{1} Z_{1} ; 2$ ) Coordinate system on fixed connection of mass center of the passive docking spacecraft: $\left.\mathrm{O}_{2} \mathrm{X}_{2} \mathrm{Y}_{2} \mathrm{Z}_{2} ; 3\right)$ Coordinate system on fixed connection with the active docking driving and guiding mechanism : $\mathrm{O}_{3} \mathrm{X}_{3} Y_{3} Z_{3}$.

All the coordinate systems are subject to right-hand rule. The transformation from the coordinate system 1 to the coordinate system 2 is performed in the following rotational order: the yaw angle $\psi$ (relative to axis $Y_{1}$ ), the pitch angle $\theta$ (relative to transition axis $Z_{12}$ ), and the rolling angle $\varphi$ (relative to axis $X_{2}$ ). The transformation matrix satisfies $\boldsymbol{A}_{21}=\boldsymbol{A}_{12}^{\mathrm{T}}$. The equation is as follows: 


$$
A_{21}=\left[\begin{array}{ccc}
\cos \psi \cos \theta & \sin \theta & -\sin \psi \cos \theta \\
\sin \varphi \sin \psi-\cos \varphi \cos \psi \sin \theta & \cos \varphi \cos \theta & \sin \varphi \cos \psi+\cos \varphi \sin \psi \sin \theta \\
\cos \varphi \sin \psi+\sin \varphi \cos \psi \sin \theta & -\sin \varphi \cos \theta & \cos \varphi \cos \psi-\sin \varphi \sin \psi \sin \theta
\end{array}\right]
$$

The transformation between the above two coordinates is expressed as:

$$
\left(\begin{array}{lll}
x_{2} & y_{2} & z_{2}
\end{array}\right)^{\mathrm{T}}=\boldsymbol{A}_{21}\left(\begin{array}{lll}
x_{1} & y_{1} & z_{1}
\end{array}\right)^{\mathrm{T}}
$$

Similarly, the transformation from the coordinate system 1 to the coordinate system 3 can be completed by matrix $\boldsymbol{A}_{13}$, the form is the same as the $\mathrm{A}_{21}$. The transformation matrix from the coordinate system 3 to the coordinate system 2 is defined as $\mathrm{A}_{32}$, hence $A_{32}=A_{31} A_{12}$. Then, the contact point $\mathrm{P}$ during the capture process in the coordinate system defined above has the following relationship as shown in Fig.2.

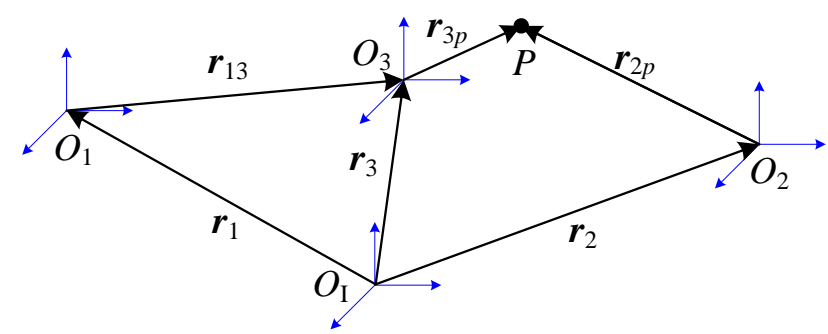

Figure 2. Docking Movement Vector Diagram

In this paper, the passive aircraft with active docking mechanism and active aircraft are considered separately, this makes it convenient for the modeling. The constraint reaction of $\boldsymbol{F}$ at the point of contact was added as external force to the passive aircraft and active docking mechanism, and the principle of virtual power is adopted to establish the dynamic equations of the docking capture process.

As shown in Fig.3, passive docking mechanism is expressed by an outer admission conical surface whose parameters can be expressed by the height $h$ of the conical surface and the angle $\beta$ of the conical surface. The position vector between the contact point $q$ and the admission cone vertex $\boldsymbol{o}_{4}$ is expressed as $\boldsymbol{r}_{4 q}$.
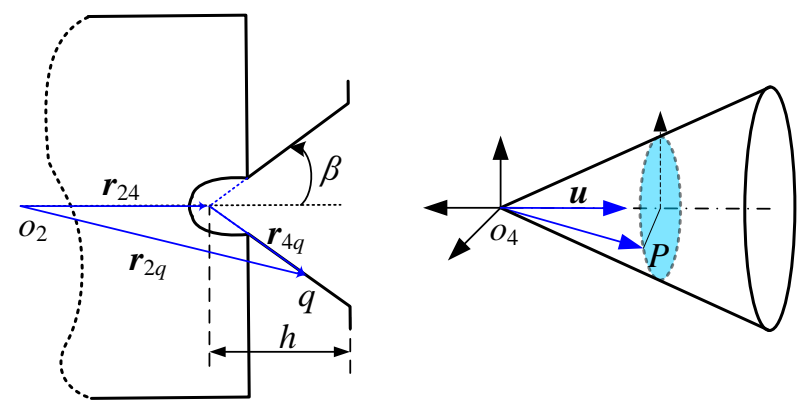

Figure 3. Parameterized Modeling of Passive Docking Mechanism

When $\beta$ is a sharp angle, it can be expressed as

$$
\left[\hat{\boldsymbol{u}} \cdot\left(\boldsymbol{x}-\boldsymbol{o}_{4}\right)\right]^{2}=\cos ^{2} \beta\left\|\boldsymbol{x}-\boldsymbol{o}_{4}\right\|^{2}
$$

Where $\hat{\boldsymbol{u}}$ is the unit vector of the symmetric axis of the conical surface.

The position vector of the contact point in the passive spacecraft is expressed as: 


$$
\boldsymbol{r}_{2 q}=\boldsymbol{r}_{24}+\boldsymbol{r}_{4 q}
$$

The inertial virtual power of the active aircraft can be described as follows:

$$
\begin{aligned}
\delta P_{1}= & \int_{c}-\ddot{\boldsymbol{r}} \cdot \delta \dot{\boldsymbol{r}} d m \\
= & -\int_{c}\left[\ddot{r}_{1}+\dot{\boldsymbol{\omega}}_{1} \times \boldsymbol{\rho}+\boldsymbol{\omega}_{1} \times\left(\boldsymbol{\omega}_{1} \times \boldsymbol{\rho}\right)\right] \cdot\left(\delta \dot{\boldsymbol{r}}_{1}+\delta \boldsymbol{\omega}_{1} \times \boldsymbol{\rho}\right) d m \\
= & -\delta \dot{\boldsymbol{r}} \cdot\left\{\int_{c}\left[\ddot{\boldsymbol{r}}_{1}+\dot{\boldsymbol{\omega}}_{1} \times \boldsymbol{\rho}+\boldsymbol{\omega}_{1} \times\left(\boldsymbol{\omega}_{1} \times \boldsymbol{\rho}\right)\right] d m\right\}-\delta \boldsymbol{\omega}_{1} \cdot\left\{\int_{c} \boldsymbol{\rho} \times\left[\ddot{\boldsymbol{r}}_{1}+\dot{\boldsymbol{\omega}}_{1} \times \boldsymbol{\rho}+\boldsymbol{\omega}_{1} \times\left(\boldsymbol{\omega}_{1} \times \boldsymbol{\rho}\right)\right] d m\right\} \\
= & -\delta \dot{\boldsymbol{r}}_{1} \cdot\left[\ddot{\boldsymbol{r}}_{1} \int_{c} d m+\dot{\boldsymbol{\omega}}_{1} \times \int_{c} \boldsymbol{\rho} d m+\boldsymbol{\omega}_{1} \times\left(\boldsymbol{\omega}_{1} \times \int_{c} \boldsymbol{\rho} d m\right)\right]- \\
& \delta \boldsymbol{\omega}_{1} \cdot\left[-\ddot{\boldsymbol{r}}_{1} \times \int_{c} \boldsymbol{\rho} d m+\int_{c}\left(\rho^{2} \boldsymbol{E}-\boldsymbol{\rho} \boldsymbol{\rho}\right) \cdot \dot{\boldsymbol{\omega}}_{1} d m+\boldsymbol{\omega}_{1} \times \int_{c}\left(\rho^{2} \ddot{\boldsymbol{E}}-\boldsymbol{\rho} \boldsymbol{\rho}\right) \cdot \boldsymbol{\omega}_{1} d m\right] \\
= & -\delta \dot{\boldsymbol{r}}_{1} \cdot\left[m_{1} \ddot{\boldsymbol{r}}_{1}-m_{1} \boldsymbol{\rho}_{1 c} \times \dot{\boldsymbol{\omega}}_{1}+m_{1} \boldsymbol{\omega}_{1} \times\left(\boldsymbol{\omega}_{1} \times \boldsymbol{\rho}_{c 1}\right)\right]-\delta \boldsymbol{\omega}_{1} \cdot\left[m_{1} \boldsymbol{\rho}_{1 c} \times \ddot{\boldsymbol{r}}_{1}+\boldsymbol{I}_{1} \cdot \dot{\boldsymbol{\omega}}_{1}+\boldsymbol{\omega}_{1} \times\left(\boldsymbol{I}_{1} \cdot \boldsymbol{\omega}_{1}\right)\right] \\
= & -\delta \dot{\boldsymbol{r}}_{1} \cdot m_{1} \ddot{\boldsymbol{r}}_{1}-\delta \boldsymbol{\omega}_{1} \cdot\left[\boldsymbol{I}_{1} \cdot \dot{\boldsymbol{\omega}}_{1}+\boldsymbol{\omega}_{1} \times\left(\boldsymbol{I}_{1} \cdot \boldsymbol{\omega}_{1}\right)\right]
\end{aligned}
$$

Where, $\boldsymbol{m}_{1}$ — quality of the tracking aircraft, $\mathrm{Kg}$.

$I_{1}$ inertia tensor of tracking aircraft on its center of mass, $\boldsymbol{I}_{1}=\int_{c}\left(\rho^{2} \boldsymbol{E}-\boldsymbol{\rho} \boldsymbol{\rho}\right) d m$.

$S_{1} \longrightarrow$ tracking aircraft static moment about fixed point, $S_{1}=\int_{c} \boldsymbol{\rho} d m=m \boldsymbol{\rho}_{1 c}$.

$\boldsymbol{E} \longrightarrow$ unit tensor.

\section{Dynamics Simulation During Capture and Separation Process}

According to the simulation model above, the kinematics and dynamics characteristics in the docking process can be analyzed based on Matlab. Considering the design of docking mechanism with axial symmetry in the structure, the position deviation, deviation of lateral position and its first derivative required by the docking initial conditions are only considered in a plane. The calculation

\begin{tabular}{|c|c|c|c|c|}
\hline Calculation condition & 1 & 2 & 3 & 4 \\
\hline $\begin{array}{l}\text { Relative closing speed (active relative to passive) } \\
\qquad v /\left(\mathrm{m} \cdot \mathrm{s}^{-1}\right)\end{array}$ & 0.05 & 0.05 & 0.05 & 0.05 \\
\hline $\begin{array}{l}\text { Lateral position deviation (active pointing to passive) } \\
\qquad \mathrm{L} / \mathrm{m}\end{array}$ & 0.1 & 0 & 0 & 0 \\
\hline $\begin{array}{l}\text { Relative deviation of the yaw angle } \\
\qquad \Delta \psi /\left(^{\circ}\right)\end{array}$ & 0 & 2.0 & 2.0 & 4.0 \\
\hline $\begin{array}{l}\text { Relative deviation of the pitch angle } \\
\qquad \Delta_{\Delta} \theta /\left({ }^{\circ}\right)\end{array}$ & 0 & 2.0 & 2.0 & 4.0 \\
\hline $\begin{array}{l}\text { Relative angular velocity } \\
\qquad \omega /\left(\bullet^{\circ} \bullet^{-1}\right)\end{array}$ & 0 & 0 & 0.8 & 0.8 \\
\hline
\end{tabular}
conditions used in the initial condition are shown in Table.2. The reason that the close flight phase is added in the simulation process is because the docking initial condition is given based on the first contact. And in the process of docking, position of the first contact point varies according to the different initial conditions. The close flight phase in the simulation of different initial conditions is designed for the first contact.

Table 2. Calculation Conditions of Initial Docking Condition

For this simulation, assume that the active aircraft in the capture process provides a thrust of $560 \mathrm{~N}$, and the quality of the active aircraft and passive aircraft are 1000 $\mathrm{kg}$ and $500 \mathrm{~kg}$ respectively. The buffer installation position in the active docking 
mechanism is of $0.29 \mathrm{~m}$, and its spring stiffness coefficient is $5 \times 10^{4} \mathrm{~N} / \mathrm{m}$. According to the four initial conditions in table 1, the capture phase analyses for the docking mechanisms are carried out using by Matlab. The simulation results of calculation condition 2 in Table. 2 are only presented as shown from Fig. 4 toFig. 6.

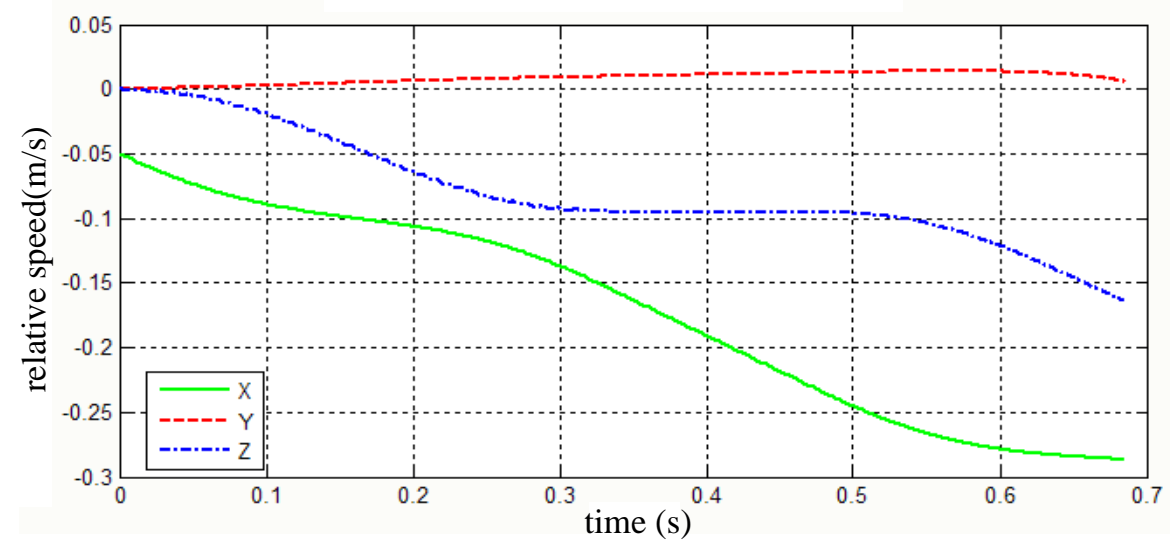

Figure 4. Speed of the Passive Spacecraft Relative to the Active Aircraft

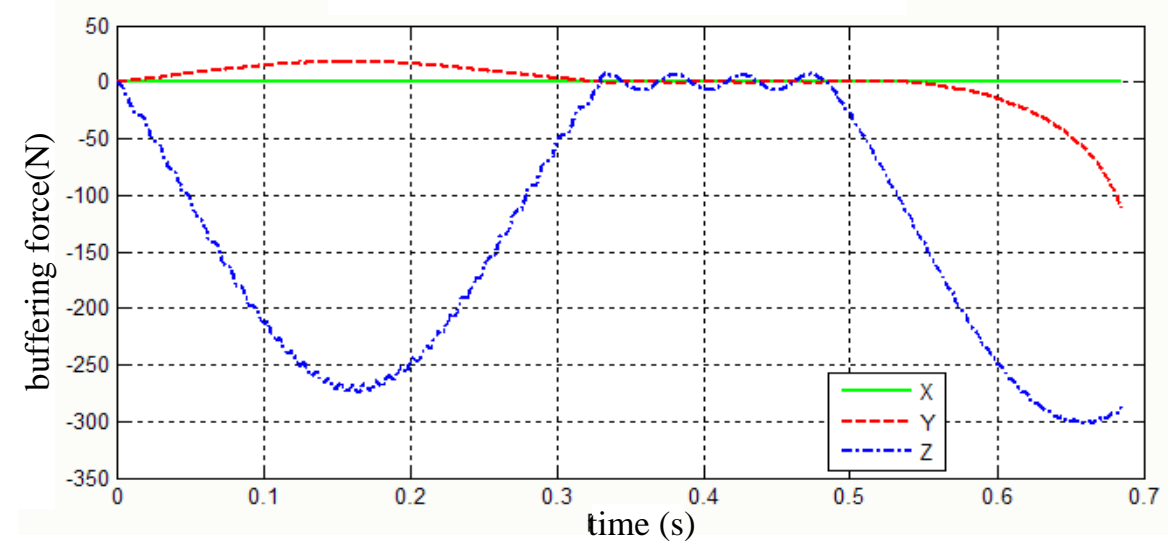

Figure 5. Change of the Buffering Force in Capture Phase

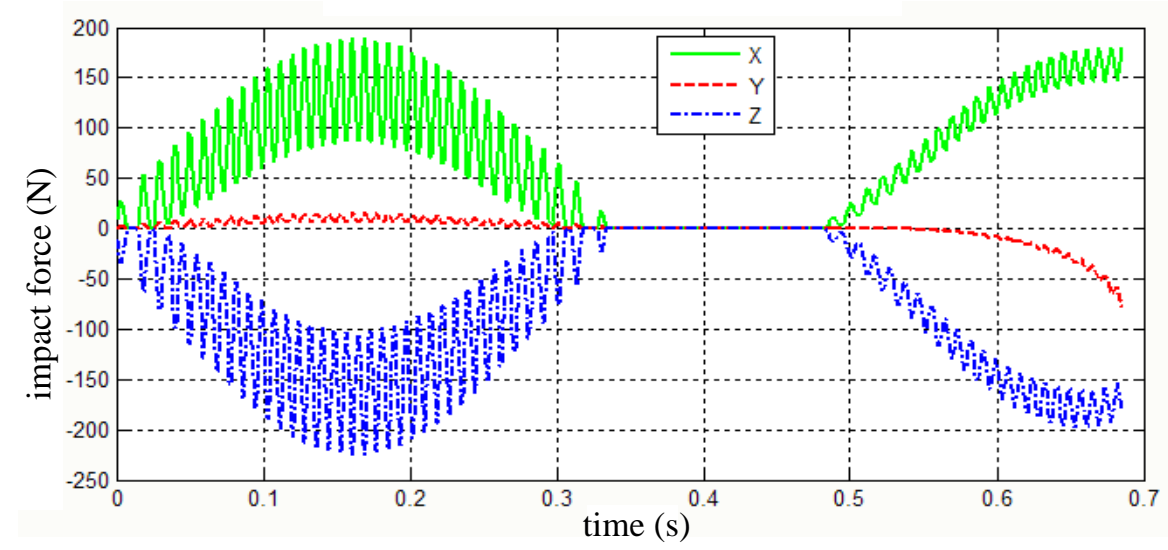

Figure 6. Change of the Impact Force in Capture Phase

The virtual prototype dynamic simulation process is controlled by ADAMS/View simulation program controller. This controller achieves the aims through controlling simulation time and step frequency. The simulation process needs to verify key parameters of the separation phase. The impact force result of the dynamic simulation during the separation phase is shown as Fig.7. 


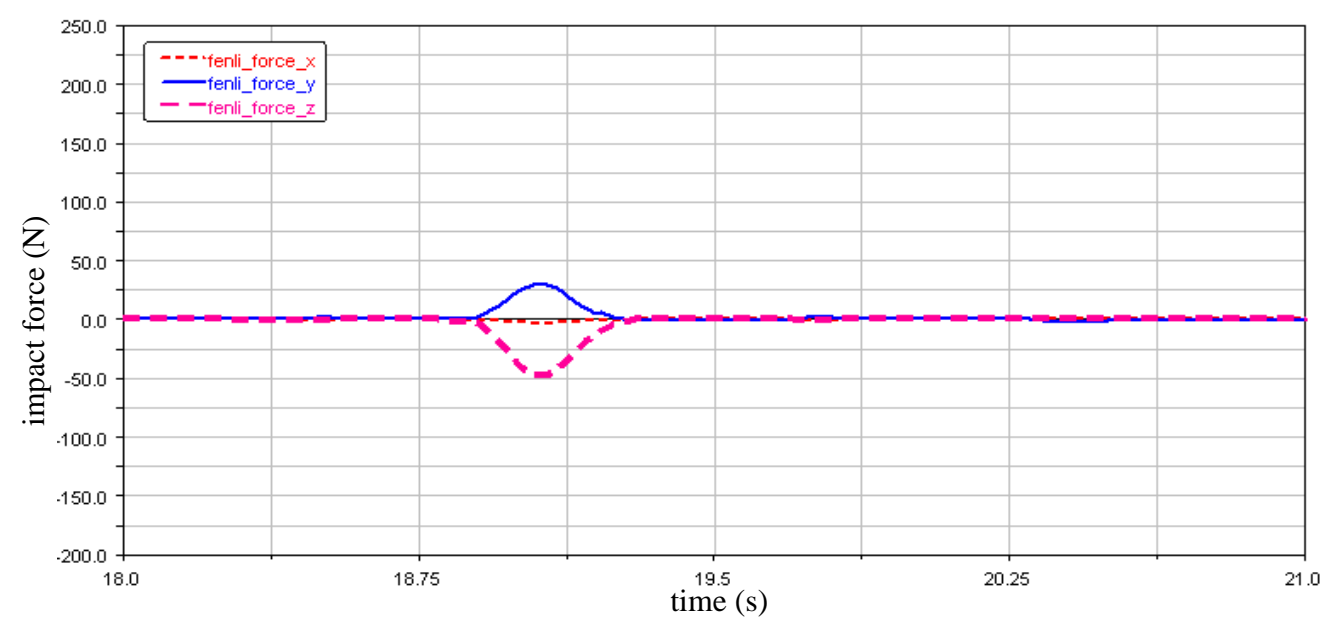

Figure 7. Impact Force in Separation Phase

It can be seen from the analysis results that the capture action between the two docking mechanisms can be completed successfully under the each of simulation. Under the condition of same thrust, the difference of capture time is not big. When the thrust is set to $560 \mathrm{~N}$, the capture time is less than $1 \mathrm{~s}$. The maximum collision force is less than $250 \mathrm{~N}$, collision force torque is less than $400 \mathrm{~N} . \mathrm{m}$; and the maximum buffering force is less than $300 \mathrm{~N}$, buffering force torque is less than 200N.m.

\section{Conclusion}

This paper gives an analysis method for researching the kinetic characteristic during the capture and separation process for a mini-type probe-cone docking device. According to the simplified structure of the docking device, the kinetic model of the docking captured separation process are established based on Matlab and Adams, and the numerical simulation is carried out at a typical operating condition with the initial lateral deviation and attitude deviation considering the change of parameters such as the magnitudes of different thrust forces. The results of kinetic analysis above suggest that the docking device have good capture performance during the capture and separation Process.

\section{Acknowledgements}

The research is supported by National Natural Science Foundation Project (51375125), Natural Science Foundation of Heilongjiang Province of China (Grant No. E201444), Natural Science Foundation of Heilongjiang Province of China (E201440).

\section{References}

[1] W. Sheng-bao, L. Mu and G. Zhao-hui, "Docking mechanism and dynamic analysis of earth-lunar orbital transfer stage”, Proceedings of the International Astronautical Congress, (2013).

[2] Z. Yuan, W. Ying-ying and Song Yan, "Kinematics analysis and simulation of small satellite docking mechanism end executor", Applied Mechanics and Materials, vol.487, (2014), pp.460-464.

[3] A. Boesso, "Francesconi. ARCADE small-scale docking mechanism for micro-satellites", Acta Astronautica, vol.86, (2013), pp.77-78.

[4] L. Ling, Y. Fei-fei and W. Yan-fang, "Finite element analysis and test of damping torque of eddy current damper for docking mechanism", Journal of Astronautics, vol. 32, no.5, (2011), pp.319-329.

[5] M. Romano, D. A. Friedman, "Laboratory experimentation of autonomous spacecraft approach and docking to a collaborative target", Journal of Spacecraft and Rockets, vol.44, no.6, (2007), pp.164-173. 
[6] Z. Xiang, H. Yi-yong and H. Wei, "Research of flexible beam impact dynamics based on space probecone docking mechanism", Advances in Space Research, vol.49, no6, (2012), pp.1053-1061.

[7] L. Yinan, D. Ye, T. Hao and Z. Dawei, "Design of an automatic autonomous mini prone-cone microsatellite docking mechanism", Chinese Journal of Mechanical Engineering (English Edition), vol.23, no.8, (2010), pp.353-360.

[8] Z. Yang, T. Hao and C. Xibin, "Effects of transmission element parameters to mechanical characteristics of docking mechanism", Aircraft Engineering and Aerospace Technology, vol.77, no.4, (2005), pp.30710 .

[9] T. Hao, Z. Yang and Z. Dawei, "Movement simulator modeling and simulation in integrate test platform for docking mechanism", Journal of Astronautics, vol.28, no.4, (2007), pp.996-1001.

[10] Z. Dawei, T. Hao and Z. Yang, "Quasi probe-cone docking mechanism capturing dynamics analyses and parameter design", Journal of Astronautics, vol.29, no.6, (2008), pp.1717-1722. 